EESTI NSV TEADUSTE AKADEEMIA TÖIMETISED.

FOUSIKA * MATEMAATIKA

ИЗВЕСТИЯ АКАДЕМИИ НАУК ЭСТОНСКОИ ССР. ФИЗИКА МАТЕМАТИКА.

PROCEEDINGS OF THE ACADEMY OF SCIENCES OF THE ESTONIAN SSR.

PHYSICS * MATHEMATICS

$1984,33,4$

П. КАРД

удК $517.94: 535.41$

\title{
МЕТОД ПОЛУЧЕНИЯ И РЕШЕНИЯ НОВЫХ ОДНОМЕРНЫХ ВОЛНОВЫХ УРАВНЕНИИ
}

В предыдущей статье $\left[{ }^{1}\right]$ был развит метод преобразования линейных дифференциальных уравнений, позволяющий получать новые уравнения, решаемые в замкнутом виде. Этот метод, в сочетании с методом статьи $\left[{ }^{2}\right]$, может быть с успехом применен к нахождению новых одномерных волновых уравнений вместе с их решениями. В настоящей статье мы излсжим соответствующую теорию и дадим затем несколько примеров.

\section{Теория}

Пусть задано имеющее известное решение волновое уравнение

$$
d^{2} U / d z^{2}+k^{2} f^{2}(z / h) U=0 .
$$

Здесь $k$ - волновое число, $z$ - координата, $h$ - постоянная размерности длины, $f(z / h)$ - показатель преломления: Покажем, каким образом можно на основе этого уравнения получить новые волновые уравнения и их решения. Для этого есть три способа.

Перепишем прежде всего уравнение (1), изменив обозначение $U \rightarrow y$ и обозначив безразмерный аргумент $z / h$ через $G$, в виде

$$
d^{2} y / d G^{2}+k^{2} h^{2} \digamma^{2}(G) y=0 .
$$

Это уравнение, согласно $\left[{ }^{1}\right]$, можно преобразовать с помощью одной из следующих трех подстановок:

$$
\begin{gathered}
\theta=f^{-1} d y / d G \\
\theta=f^{-1}\left(\frac{d y}{d G}-\frac{M y}{M G+N}\right)
\end{gathered}
$$

( $M$ и $N$ - произвольные постоянные) и

$$
\theta=f^{-1}\left(d y / d G-u^{-1} y d u / d G\right) .
$$

В последней формуле $u$-любая функция, удовлетворяющая уравнению

$$
d^{2} u / d G^{2}+C^{2} \digamma^{2}(G) u=0,
$$

где $C \neq 0$ - произвольная постояннал. Отметим, что решение последнего уравнения известно, так как известно решение уравнения (2).

Применив все три подстановки к уравнению (2), науодим следующие три уравнения для $\theta$ :

1) в случае подстановки (3)

$$
d^{2} \theta / d G^{2}+\left(k^{2} h^{2} f^{2}-2 f^{-2} f^{\prime 2}+f^{-1} f^{\prime \prime}\right) \theta=0 ;
$$

2) в случае подстановки (4)

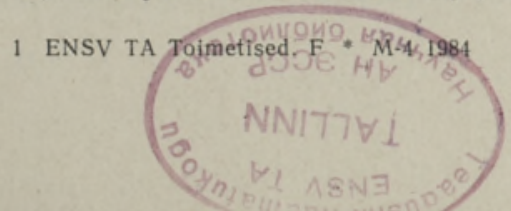


$d^{2} \theta / d G^{2}+\left[k^{2} h^{2} f^{2}-2 f^{-2} f^{\prime 2}+f^{-1} f^{\prime \prime}-\frac{2 M f^{-1} f^{\prime}}{M G+N}-\frac{2 M^{2}}{(M G+N)^{2}}\right] \theta=0$

3) в случае подстановки (5)

$d^{2} \theta / d G^{2}+\left(k^{2} h^{2} f^{2}-2 f^{-2} f^{\prime 2}+f^{-1} f^{\prime \prime}-2 f^{-1} f^{\prime} u^{-1} u^{\prime}-2 u^{-2} u^{\prime 2}-2 C^{2} f^{2}\right) \theta=0$.

Во всех этих уравнениях штрих означает производную по $G$. Решения всех трех уравнений известны; они даются формулами (3)-(5), где y и $u$ - известные функции.

Нашей целью является, однако, получение и решение новых волновых уравнений. Этой цели служит метод статьи $\left[{ }^{2}\right]$. Согласно этому методу мы можем поставить каждому из уравнений (7)-(9) в соответствие волновое уравнение вида

$$
d^{2} U / d z^{2}+k^{2}(c z / h+d)^{-4} f^{2}(G) \Psi^{4}(G) U=0 .
$$

Решение этого уравнения дается формулой

$$
U=(c z / h+d) \Psi^{-1} \theta,
$$

причем функция $\Psi$ должна удовлетворять такому же уравнению, что и $\theta$, но при $k=0$. Выбор функции $\Psi$ ничем иным не ограничен, так как параметр $G$ связан с координатой $z$ формулой

$$
\int \Psi^{-2} d G=\frac{a z / h+b}{c z / h+d}, \quad a d-b c=1,
$$

откуда видно, что переход к другой функции $\Psi$ означает просто соответствующую замену постоянных $a, b, c, d$.

Если $k=0$, то уравнение (2) имеет решение $y=A G$, где $A-$ постоянная. Следовательно, подставляя в правые части формул (3)-(5) $A G$ вместо $y$, найдем в левых частях $\Psi$ вместо $\theta$. Возьмем в формуле (3) $y=G$, в формуле (4) $y=N^{-1} G$ и в формуле (5) $y=C^{-1} G$. Тогда получим, соответственно,

$$
\begin{gathered}
\Psi=f^{-1}, \\
\Psi=f^{-1}(M G+N)^{-1}
\end{gathered}
$$

и

$$
\Psi=C^{-1} f^{-1}\left(1-G u^{-1} u^{\prime}\right) .
$$

Отсюда формула (12) в тех же трех случаях дает

$$
\begin{gathered}
\frac{a z / h+b}{c z / h+d}=\int f^{2}(G) d G \\
\frac{a z / h+b}{c z / h+d}=\int f^{2}(G)(M G+N)^{2} d G
\end{gathered}
$$

и

$$
\frac{a z / h+b}{c z / h+d}=\left(G-u u^{\prime-1}\right)^{-1}
$$

Волновое уравнение (10) получается тоже в трех видах:

$$
\begin{gathered}
d^{2} U / d z^{2}+k^{2}(c z / h+d)^{-4} f^{-2}(G) U=0, \\
d^{2} U / d z^{2}+k^{2}(c z / h+d)^{-4} f^{-2}(G)(M G+N)^{-4} U=0
\end{gathered}
$$




$$
d^{2} U / d z^{2}+k^{2} C^{-4}(c z / h+d)^{-4} f^{-2}(G)\left(1-G u^{-1} u^{\prime}\right)^{4} U=0 .
$$

Решения этих уравнений, согласно формулам (11), (3)-(5) и (13)(15), таковы:

$$
\begin{gathered}
U=(c z / h+d) d y / d G, \\
U=(c z / h+d)[(M G+N) d y / d G-M y]
\end{gathered}
$$

и

$$
U=(c z / h+d)\left(1-G u^{-1} d u / d G\right)^{-1}\left(d y / d G-u^{-1} y d u / d G\right) .
$$

Итак, исходя из волнового уравнения (1), решение которого предполагаем известным, мы получили три новых волновых уравнения (19)(21) с их решениями $(22)-(24)$. Уравнение (21) содержит в себе даже множество уравнений, так как функция $u$, удовлетворяющая уравнению (6), может быть выбрана различным образом. Нужно, однако, отметить, что далеко не всегда уравнения (19)-(21) получаются новыми; нередко они оказываются уже известными и может даже случиться, что воспроизводится исходное уравнение (1). Параметр $G$ далеко не всегда поддается исключению, и часто приходится удовлетворяться параметрической формой уравнения. Тем не менее изложенный метод является достаточно плодотворным, о чем свидетельствуют приводимые ниже примеры.

\section{Примеры}

В примерах мы не будем приводить всех выкладок, а только будем указысать исходное уравнение, его решение, род преобразования и конечныі результат, т. е. новое волновое уравнение и его решение. Для сокращения записи будем пользоваться обозначением

$$
\xi=\frac{a z / h+b}{c z / h+d}, \quad a d-b c=1 .
$$

Начнем с уравнения

$$
d^{2} y / d G^{2}+k^{2} h^{2}\left(A^{2} G^{m}+B^{2} G^{-2}\right) y=0, \quad A \neq 0, \quad m \neq-2,
$$

имеющего решение

$$
y(G)=G^{1 / 2} Z_{v}\left(\frac{2 k h A}{m+2} G^{\frac{m+2}{2}}\right),
$$

где $Z_{v}$ - функция Бесселя (цилиндрическая функция) порядка

$$
v=\frac{\left(1-4 k^{2} h^{2} B^{2}\right)^{1 / 2}}{|m+2|}
$$

Преобразование вида (3) дает согласно формуле (16)

$$
\xi=\frac{A^{2} G^{m+1}}{m+1}-B^{2} G^{-1}, \quad m \neq-1
$$

и, если $m=-1$,

$$
\xi=A^{2} \ln G-B^{2} G^{-1} .
$$

Эти формулы связывают параметр $G$ с координатой $z$. Новое волновое уравнение, согласно формуле (19), есть 


$$
d^{2} U / d z^{2}+k^{2}(c z / h+d)^{-4}\left(A^{2} G^{m}+\hat{B}^{2} G^{-2}\right)^{-1} U=0,
$$

а его решение, согласно формулам (22) и (27), имеет вид

$$
\begin{aligned}
U & =(c z / h+d)\left\{G^{-1 / 2} Z_{v}\left(\frac{2 k h A}{m+2} G^{\frac{m+2}{2}}\right)+\right. \\
& \left.+2 k h A G^{\frac{m+1}{2}} Z_{v}^{\prime}\left(\frac{2 k h A}{m+2} G^{\frac{m+2}{2}}\right)\right\},
\end{aligned}
$$

где штрих у $Z_{v}$ означает производную по аргументу.

Рассмотрим подробнее несколько простейших случаев, в которых параметр исключается. Будем считать $B \neq 0$, так как при $B=0$ получаются уже известные уравнения. Чтобы уравнение (29) решалось в общем виде относительно $G$, оно должно быть степени не выше четвертой. Этому условию удовлетворяют значения $m 0,-3 / 2,-3$, дающие квадратное уравнение, $1,-1 / 2,-4 / 3,-5 / 3,-5 / 2,-4$, дающие уравнение третьей степени, и $2,-2 / 3,-5 / 4,-7 / 4,-7 / 3,-5$, дающие уравнение четвертой степени. Все эти уравнения решаются в радикалах, однако мы ограничимся только случаями квадратных уравнений, как наиболее простых.

I. Если $m=0$, то, заменив постоянные $A \rightarrow A^{-1}$ и $B \rightarrow A B / 2$, приходим к волновому уравнению

$$
d^{2} U / d z^{2}+\frac{k^{2} A^{2}}{2}(c z / h+d)^{-4}\left(1 \pm \frac{\xi}{\sqrt{\xi^{2}+B^{2}}}\right) U=0
$$

решение которого имеет вид

$$
\begin{aligned}
& U(z)=(c z / h+d)\left\{\left(\sqrt{\xi^{2}+B^{2}} \mp \xi\right)^{1 / 2} Z_{\frac{1}{2}\left(1-A^{2} B^{2} k^{2} h^{2}\right)^{1 / 2}}\left(\frac{k h A}{2}\left(\sqrt{\xi^{2}+B^{2}} \pm \xi\right)\right)+\right. \\
& \left.+A B k h\left(\sqrt{\xi^{2}+B^{2}} \pm \xi\right)^{1 / 2} Z^{\prime}{ }_{\frac{1}{2}\left(1-A^{2} B^{2} k^{2} h^{2}\right)^{1 / 2}}\left(\frac{k h A}{2}\left(\sqrt{\xi^{2}+B^{2}} \pm \xi\right)\right)\right\}
\end{aligned}
$$

Здесь $A$ и $B$ могут иметь не только вещественные, но и мнимые значения; однако, при мнимом $B$ коэффициент при $U$ в уравнении положителен только с нижним знаком, если и $A$ мнимо, и с верхним знаком, если A вещественно.

II. Если $m=-3 / 2$, то, заменив постоянную $A \rightarrow i(-A)^{-1 / 4} B^{1 / 2}$, находим волновое уравнение

$$
d^{2} U / d z^{2}+\frac{k^{2} A^{2} B^{2}(c z / h+d)^{-4} U}{\sqrt{1+A \xi}(1+\sqrt{1+A \xi})^{3}}=0
$$

с решением

$$
\begin{aligned}
U(z) & =(c z / h+d)\left\{(1+\sqrt{1+A \xi}) Z_{2\left(1-4 k^{2} h^{2} B^{2}\right)^{1 / 2}}\left(\frac{4 i k h B}{\sqrt{1+\sqrt{1+A \xi}}}\right)+\right. \\
& \left.+2 i k h B \sqrt{1+\sqrt{1+A \xi}} Z_{2\left(1-4 k^{2} h^{2} B^{2}\right)^{1 / 2}}^{\prime}\left(\frac{4 i k h B}{\sqrt{1+\sqrt{1+A \xi}}}\right)\right\}
\end{aligned}
$$

Это уравнение можно несколько видоизменить, взяв перед корнем обратный знак и заменив $A \rightarrow-A$ и $B \rightarrow i B$. Тогда получим уравнение 


$$
d^{2} U / d z^{2}+\frac{k^{2} A^{2} B^{2}(c z / h+d)^{-4} U}{\sqrt{1-A \xi}(1-\sqrt{1-A \xi})^{3}}=0
$$

с решением

$$
\begin{aligned}
& U(z)=(c z / h+d)\left\{(\cdot 1-\sqrt{1-A \xi}) Z_{2\left(1+4 k^{2} h^{2} B^{2}\right)^{1 / 2}}\left(\frac{4 k h B}{\sqrt{1-\sqrt{1-A \xi}}}\right)+\right. \\
& \left.+2 k h B \sqrt{1-\sqrt{1-A \xi}} Z_{2\left(1+4 k^{2} h^{2} B^{2}\right)^{1 / 2}}^{\prime}\left(\frac{4 k h B}{\sqrt{1-\sqrt{1-A \xi}}}\right)\right\} .
\end{aligned}
$$

III. Если $m=-3$, то, заменив постоянную $A \rightarrow 2^{-1 / 2}(-A)^{1 / 2} B^{2}$, находим волновое уравнение

$$
d^{2} U / d z^{2}+\frac{k^{2} A^{2} B^{2}(c z / h+d)^{-4} U}{4 \sqrt{1+A \xi}(-1+\sqrt{1+A \xi})^{2}}=0
$$

с решением

$$
\begin{aligned}
U(z) & =(c z / h+d)\left\{(-1+\sqrt{1+A \xi})^{1 / 2} Z_{\left(1-4 k^{2} h^{2} B^{2}\right)^{1 / 2}}(2 k h B \sqrt{-1+\sqrt{1+A \xi}})-\right. \\
& \left.-2 k h B(-1+\sqrt{1+A \xi}) Z_{\left(1-4 k^{2} h^{2} B^{2}\right)^{1 / 2}}^{\prime}(2 k h B \sqrt{-1+\sqrt{1+A \xi}})\right\}
\end{aligned}
$$

Итак, уравнение (26) дает три новых решаемых в замкнутом виде волновых уравнения (не считая вариантов) с исключенным параметром - (33), (35) или (37) и (40). Перейдем к другим примерам.

IV. Новое уравнение с исключенным параметром получим, исходя из уравнения (39). Перепишем его в виде

$$
d^{2} y / d G^{2}+\frac{k^{2} h^{2} A^{2} B^{2} y}{4 \sqrt{1+A G}(-1+\sqrt{1+A G})^{2}}=0
$$

и применим преобразование (4) с $M=1$ и $N=0$. Формула (17) дает

$$
\xi=\left(B^{2} / 6 A\right)(1+\sqrt{1+A G})^{3} .
$$

Заменив здесь для простоты $6 A B^{-2} \xi \rightarrow \xi$, находим по исключении параметра волновое уравнение

$$
d^{2} U / d z^{2}+\left(k^{2} B^{2} / 9\right)(c z / h+d)^{-4}\left(\xi^{1 / 3}-1\right)\left(\xi-2 \xi^{2 / 3}\right)^{-2} U=0
$$

и его решение

$$
\begin{gathered}
U(z)=(c z / h+d)\left\{\left(\xi^{1 / 3}-2\right)^{1 / 2}\left(k^{2} h^{2} B^{2} \xi^{1 / 3}-1\right) Z_{\left(1-4 k^{2} h^{2} B^{2}\right)^{1 / 2}}\left(2 k h B\left(\xi^{1 / 3}-2\right)^{1 / 2}\right)+\right. \\
\left.+2 k h B\left(\xi^{1 / 3}-2\right) Z_{\left(1-4 k^{2} h^{2} B^{2}\right)^{1 / 2}}^{\prime}\left(2 k h B\left(\xi^{1 / 3}-2\right)^{1 / 2}\right)\right\} .
\end{gathered}
$$

Из большого числа волновых уравнений параметрического вида, которые могут быть выведены нашим методом, приведем только два.

$\mathrm{V}$. Возьмем уравнение

$$
d^{2} y / d G^{2}+k^{2} h^{2} A^{2}\left(1+G^{2}\right)^{-2} \arctan G \cdot y=0,
$$

имеющее решение (см. $\left.\left[{ }^{3}\right]\right)$ 


$$
\begin{gathered}
y=\left(1+G^{2}\right)^{1 / 2}\left(1+k^{2} h^{2} A^{2} \arctan G\right)^{1 / 2} \times \\
\times Z_{1 / 3}\left(\left(2 / 3 k^{2} h^{2} A^{2}\right)\left(1+k^{2} h^{2} A^{2} \arctan G\right)^{3 / 2}\right) .
\end{gathered}
$$

Применив преобразование (3), приходим к волновому уравнению

$$
d^{2} U / d z^{2}+k^{2} A^{2}(c z / h+d)^{-4} \arctan ^{-1} G\left(1+G^{2}\right)^{2} U=0,
$$

решение которого имеет вид

$$
\begin{gathered}
U=(c z / h+d)\left(1+G^{2}\right)^{-1 / 2}\left\{\left[G\left(1+k^{2} h^{2} A^{2} \arctan G\right)^{1 / 2}+\right.\right. \\
\left.+\left(k^{2} h^{2} A^{2} / 2\right)\left(1+k^{2} h^{2} A^{2} \arctan G\right)^{-1 / 2}\right] \times \\
\times Z_{1 / 3}\left(\left(2 / 3 k^{2} h^{2} A^{2}\right)\left(1+k^{2} h^{2} A^{2} \arctan G\right)^{3 / 2}\right)+ \\
\left.+\left(1+k^{2} h^{2} A^{2} \arctan G\right) Z_{1 / 3}^{\prime}\left(\left(2 / 3 k^{2} h^{2} A^{2}\right)\left(1+k^{2} h^{2} A^{2} \arctan G\right)^{3 / 2}\right)\right\}
\end{gathered}
$$

причем параметр связан с координатой формулой

$$
\frac{a z / h+b}{c z / h+d}=\frac{\arctan ^{2} G}{4}+\frac{G \arctan G}{2\left(1+G^{2}\right)}+\frac{1}{4\left(1+G^{2}\right)} .
$$

VI. В последнем примере используем преобразование (5). За исходное уравнение примем

$$
d^{2} y / d G^{2}+k^{2} h^{2} A^{2} G y=0,
$$

имеющее решение

$$
y=G^{1 / 2} Z_{1 / 3}\left(\frac{2 k h A G^{3 / 2}}{3}\right)
$$

Соответственно, уравнение

$$
d^{2} u / d G^{2}+C^{2} A^{2} G u=0
$$

имеет решение

$$
u=G^{1 / 2} Z_{1 / 3}\left(\frac{2 C A G^{3 / 2}}{3}\right)
$$

Подставляя эти функции в формулы (18), (21) и (24), находим в результате довольно громоздких выкладок и надлежащих упрощений волновое уравнение

$$
d^{2} U / d z^{2}+\left(k^{2} C^{2} / 36\right)(c z / h+d)^{-4} G^{-2 / 3}\left(1-3 G Z_{1 / 3}^{\prime}(G) / Z_{1 / 3}(G)\right)^{4} U=0
$$

и его решение

$$
\begin{gathered}
U=(c z / h+d) G^{2 / 3}\left(Z_{1 / 3}(G)-3 G Z_{1 / 3}^{\prime}(G)\right)^{-1} \times \\
\times\left(k h C Z_{1 / 3}(G) Z_{1 / 3}^{\prime}(k h C G)-Z_{1 / 3}^{\prime}(G) Z_{1 / 3}(k h C G)\right),
\end{gathered}
$$

причем

$$
\frac{a z / h+b}{c z / h+d}=\frac{Z_{1 / 3}(G)+3 G Z_{1 / 3}^{\prime}(G)}{G^{2 / 3}\left(Z_{1 / 3}(G)-3 G Z_{1 / 3}^{\prime}(G)\right)} .
$$

\section{Л И ТЕ Р А Т У Р А}

1. Кард П. Изв. АН ЭССР. Физ. Матем., 33, № 3, 261-265 (1984).

2. Кард П. Изв. АН ЭССР. Физ. Матем., 33, № 2, 137-146 (1984).

3. Кард П. Изв. АН ЭССР. Физ. Матем., 29, № 1, 1-7 (1980). 


\section{UUTE UHE:MOOTMELISTE LAINEVORRANDITE \\ JA NENDE LAHENDITE LEIDMISE MEETOD}

Asendused (3), (4) või (5), kus $u$ on võrrandi (6) lahend, teisendavad tuntud lahendiga võrrandi (2) võrranditeks (7), (8) või (9). Nendest vốrranditest järelduvad lainevôrrandid (19), (20) või (21), mille lahendid on (22), (23) vôi (24), kusjuures parameetri $G$ ja koordinaadi $z$ vahelised seosed on (16), (17) või (18). Sel meetodil on saadud uued lainevōrrandid (33), (35), (37), (39) ja (43), mille lahendid on (34), (36), (38), (40) ja (44); lisaks lainevôrrandid parameetrilises kujus (31), (47) ja (54), mille lahendid on (32), (48) ja (55), kusjuures parameetri ja koordinaadi vahelised seosed on (29) või (30), (49) ia (56).

\section{P. KARD}

\section{A METHOD FOR GETTING NEW ONE-DIMENSIONAL WAVE EQUATIONS WITH THEIR SOLUTIONS}

The substitutions (3), (4) or (5), where $u$ is a solution of the equation (6). transform the equation (2) having known solution into the equations (7), (8) or (9). From these equations follow new one-dimensional wave equations (19), (20) or (21), whose solutions are (22), (23) or (24), respectively, the parameter $G$ being. connected with the co-ordinate $z$ via the formulae (16), (17) or (18). By this method an amount of new wave equations is obtained, viz. equations (33), (35), (37), (39), and (43), whose solutions are (34), (36), (38), (40), and (44), respectively, besides parametrical equations (31), (47), and (54), whose solutions are (32), (48), and (55), the parąmeter being connected with the co-ordinate via the formulae (29) (or (30)), (49), and (56). 